\title{
On the quark component in prompt photon and electroweak gauge boson production at high energies
}

\author{
A.V. Lipatov, N.P. Zotov \\ November 4, 2018 \\ D. V. Skobeltsyn Institute of Nuclear Physics, \\ M. V. Lomonosov Moscow State University, \\ 119991 Moscow, Russia
}

\begin{abstract}
In the framework of the $k_{T}$-factorization approach, we study the production of prompt photons and electroweak gauge bosons in high energy proton-(anti)proton collisions at modern colliders. Our consideration is based on the amplitude for the production of a single photon or $W^{ \pm} / Z^{0}$ boson associated with a quark pair in the fusion of two off-shell gluons. The quark component is taken into account separately using the quark-gluon scaterring and quark-antiquark annihilation QCD subprocesses. Special attention is put on the contributions from the quarks involved into the earlier steps of the evolution cascade. Using the Kimber-Martin-Ryskin formalism, we simulate this component and demonstrate that it plays an important role at both the Tevatron and LHC energies. Our theoretical results are compared with recent experimental data taken by the D $\oslash$ and CDF collaborations at the Tevatron.
\end{abstract}

PACS number(s): 12.38.-t, 12.38.Bx

\section{Introduction}

The theoretical and experimental studying the prompt photon and electroweak gauge boson production at high energies provide an important information about the nature of both the underlying electroweak interaction and the effects of Quantum Chromodynamics (QCD). In many respects these processes have become one of most important "standard candles" in experimental high energy physics [1-11].

In the previous publications [12-14], we have considered the production of prompt photons and electroweak gauge bosons $W^{ \pm}$and $Z^{0}$ in the $k_{t}$-factorization approach. Making 
use of the $k_{T}$-factorization is motivated by the fact that it provides solid theoretical grounds for the effects of initial gluon radiation and intrinsic parton transverse momentum $k_{T}$. We pay attention to individual contributions from the different partonic subprocesses. The idea of $[12,14]$ was in reexpressing the quark contributions in terms of gluon contributions, thus reducing the problem of poorly known and poorly calculable unintegrated quark densities to much better investigated gluon ones. Our studies, however, reveal the fact that the nonreducible quark distributions are of major importance for the processes under study. The goal of this paper is to clarify this point in more detail and to accomplish the calculations presented in $[12,14]$ by including the contributions which yet have not been taken into account.

The outline of our paper is following. In Section 2 we recall shortly the basic formulas of the $k_{T}$-factorization approach with a brief review of calculation steps. In Section 3 we present the numerical results of our calculations. The central point is discussing the role of each contribution to the cross sections. Section 4 contains our conclusions.

\section{Theoretical framework}

\subsection{The subprocesses under consideration}

Our approach is the following. The starting point of consideration is the leading order $\mathcal{O}(\alpha)$ and $\mathcal{O}\left(\alpha \alpha_{s}\right)$ subprocesses: $q+g^{*} \rightarrow \gamma+q, q+\bar{q} \rightarrow \gamma+g$ and $q+\bar{q}^{\prime} \rightarrow W^{ \pm} / Z^{0}$. These subprocesses are strongly depend on the unintegrated quark distributions in a proton $f_{q}\left(x, \mathbf{k}_{T}^{2}, \mu^{2}\right)$. In contrast to the study [15] where the hard matrix elements of these subprocesses have been convoluted with the relevant unintegrated quark and/or gluon distributions in a proton, we try to reexpress the unintegrated quark densities in terms of gluon ones. Our main idea is connected with the separation of the unintegrated quark distributions into several parts which correspond to the interactions of valence quarks $f_{q}^{(v)}\left(x, \mathbf{k}_{T}^{2}, \mu^{2}\right)$, sea quarks appearing at the last step of the gluon evolution $f_{q}^{(g)}\left(x, \mathbf{k}_{T}^{2}, \mu^{2}\right)$ and sea quarks coming from the earlier gluon splittings $f_{q}^{(s)}\left(x, \mathbf{k}_{T}^{2}, \mu^{2}\right)$ (see Fig. 1). In our approach, we simulate the last gluon splittings by the higher-order $\mathcal{O}\left(\alpha \alpha_{s}^{2}\right)$ off-shell (i.e. $k_{T}$-dependent) matrix elements, namely $g^{*}+g^{*} \rightarrow \gamma / W^{ \pm} / Z^{0}+q+\bar{q}^{\prime}$. In this way we take into account the contributions from the $f_{q}^{(g)}\left(x, \mathbf{k}_{T}^{2}, \mu^{2}\right)$. To estimate the contributions from the $f_{q}^{(v)}\left(x, \mathbf{k}_{T}^{2}, \mu^{2}\right)$ and $f_{q}^{(s)}\left(x, \mathbf{k}_{T}^{2}, \mu^{2}\right)$ we use the specific properties of the Kimber-Martin-Ryskin (KMR) scheme [16] which enables us to discriminate between the various components of the unintegrated quark densities 1 . Thus, the proposed scheme results to the following partonic subprocesses:

$$
\begin{gathered}
g^{*}+g^{*} \rightarrow \gamma / W^{ \pm} / Z^{0}+q+\bar{q}^{\prime}, \\
q^{(v)}+g^{*} \rightarrow \gamma / W^{ \pm} / Z^{0}+q^{\prime}, \\
q^{(s)}+g^{*} \rightarrow \gamma / W^{ \pm} / Z^{0}+q^{\prime}, \\
q+\bar{q} \rightarrow \gamma+g, \quad q+\bar{q}^{\prime} \rightarrow W^{ \pm} / Z^{0} .
\end{gathered}
$$

To be precise, the gluon-gluon fusion subprocess (1) replaces the $q^{(g)}+\bar{q}^{(g)}$ annihilation, and the valence and sea quark-gluon scattering (2) and (3) replace the $q^{(v)}+\bar{q}^{(g)}$ and $q^{(s)}+\bar{q}^{(g)}$

\footnotetext{
${ }^{1}$ Below we will refer to the $f_{q}^{(s)}\left(x, \mathbf{k}_{T}^{2}, \mu^{2}\right)$ contribution as to "reduced sea" component.
} 
annihilation mechanisms. In the last two cases both valence $q^{(v)}$ and "reduced sea" $q^{(s)}$ quark components are included. Of course, all incoming gluons in (1) - (4) are off-shell. To avoid the double counting we have not considered here $q+\bar{q}^{\prime} \rightarrow W^{ \pm} / Z^{0}+g$ subprocess.

As it was mentioned above, the proposed scheme was applied already to the prompt photon [12] and electroweak boson [14] production at the Tevatron and LHC energies. However, in [12] we have neglected the "reduced sea" contribution. In [14], to estimate the "reduced sea" component only the contribution from the $q^{(s)}+\bar{q}^{(v)} \rightarrow W^{ \pm} / Z^{0}$ subprocess has been taken into account since this contribution is the dominant one at the Tevatron energies (see also discussion in Section 3). In the present study we give a more accurate analysis of all possible contributions to the cross sections of processes under consideration 2 and clarify the role of missing contributions to the "reduced sea" component at the LHC.

\subsection{Cross section for the inclusive $\gamma / W^{ \pm} / Z^{0}$ production}

To calculate the cross section of the prompt photon and/or electroweak boson production in the framework of the $k_{T}$-factorization approach one should convolute the off-shell matrix elements of subprocesses (1) — (4) with the relevant unintegrated quark and/or gluon distributions. The contribution to the inclusive $\gamma / W^{ \pm} / Z^{0}$ production cross section from the off-shell gluon-gluon fusion (1) can be written as

$$
\begin{aligned}
& \sigma\left(p+\bar{p} \rightarrow \gamma / W^{ \pm} / Z^{0}+X\right)=\sum_{q} \int \frac{\left|\overline{\mathcal{M}}\left(g^{*}+g^{*} \rightarrow \gamma / W^{ \pm} / Z^{0}+q+\bar{q}^{\prime}\right)\right|^{2}}{256 \pi^{3}\left(x_{1} x_{2} s\right)^{2}} \times \\
& \times f_{g}\left(x_{1}, \mathbf{k}_{1 T}^{2}, \mu^{2}\right) f_{g}\left(x_{2}, \mathbf{k}_{2 T}^{2}, \mu^{2}\right) d \mathbf{k}_{1 T}^{2} d \mathbf{k}_{2 T}^{2} d \mathbf{p}_{1 T}^{2} \mathbf{p}_{2 T}^{2} d y d y_{1} d y_{2} \frac{d \phi_{1}}{2 \pi} \frac{d \phi_{2}}{2 \pi} \frac{d \psi_{1}}{2 \pi} \frac{d \psi_{2}}{2 \pi}
\end{aligned}
$$

where $\left|\overline{\mathcal{M}}\left(g^{*}+g^{*} \rightarrow \gamma / W^{ \pm} / Z^{0}+q+\bar{q}^{\prime}\right)\right|^{2}$ is the off-mass shell matrix element squared (and averaged over the initial gluon polarizations and colors); $\sqrt{s}$ is the total energy of the process under consideration; $\mathbf{k}_{1 T}, \mathbf{k}_{2 T}, \phi_{1}$ and $\phi_{2}$ are the transverse momenta and azimuthal angles of the initial off-shell gluons (having the fractions $x_{1}$ and $x_{2}$ of the incoming protons longitudinal momenta); $\mathbf{p}_{T}$ and $y$ are the transverse momentum and rapidity of the produced prompt photon or vector boson; $\mathbf{p}_{1 T}$ and $\mathbf{p}_{2 T}$ the transverse momenta of the co-produced quark and antiquark; $y_{1}, y_{2}, \psi_{1}$ and $\psi_{2}$ are the quark rapidities and azimuthal angles, respectively. The formulas for the partonic subprocesses (2) — (4) are similar and can be written as follows:

$$
\begin{gathered}
\sigma\left(p+\bar{p} \rightarrow \gamma / W^{ \pm} / Z^{0}+X\right)=\sum_{q} \int \frac{\left|\overline{\mathcal{M}}\left(q+g^{*} \rightarrow \gamma / W^{ \pm} / Z^{0}+q^{\prime}\right)\right|^{2}}{16 \pi\left(x_{1} x_{2} s\right)^{2}} \times \\
\times f_{q}\left(x_{1}, \mathbf{k}_{1 T}^{2}, \mu^{2}\right) f_{g}\left(x_{2}, \mathbf{k}_{2 T}^{2}, \mu^{2}\right) d \mathbf{k}_{1 T}^{2} d \mathbf{k}_{2 T}^{2} d \mathbf{p}_{T}^{2} d y d y^{\prime} \frac{d \phi_{1}}{2 \pi} \frac{d \phi_{2}}{2 \pi} \\
\sigma(p+\bar{p} \rightarrow \gamma+X)=\sum_{q} \int \frac{\mid \overline{\mathcal{M}}\left(q+\bar{q} \rightarrow \gamma+\left.g\right|^{2}\right.}{16 \pi\left(x_{1} x_{2} s\right)^{2}} \times \\
\times f_{q}\left(x_{1}, \mathbf{k}_{1 T}^{2}, \mu^{2}\right) f_{q}\left(x_{2}, \mathbf{k}_{2 T}^{2}, \mu^{2}\right) d \mathbf{k}_{1 T}^{2} d \mathbf{k}_{2 T}^{2} d \mathbf{p}_{T}^{2} d y d y_{g} \frac{d \phi_{1}}{2 \pi} \frac{d \phi_{2}}{2 \pi}
\end{gathered}
$$

\footnotetext{
${ }^{2}$ In the calculations of the prompt photon cross sections we will neglect the contributions from the so-called fragmentation mechanism [17]. It is because after applying the isolation cut (see [7-11]) these contributions amount only to about $10 \%$ of the visible cross section. The isolation requirement and additional conditions which preserve our calculations from divergences have been specially discussed in $[12,13]$.
} 


$$
\begin{aligned}
\sigma(p+\bar{p} & \left.\rightarrow W^{ \pm} / Z^{0}+X\right)=\sum_{q} \int \frac{2 \pi}{\left(x_{1} x_{2} s\right)^{2}}\left|\overline{\mathcal{M}}\left(q+\bar{q}^{\prime} \rightarrow W^{ \pm} / Z^{0}\right)\right|^{2} \times \\
& \times f_{q}\left(x_{1}, \mathbf{k}_{1 T}^{2}, \mu^{2}\right) f_{q}\left(x_{2}, \mathbf{k}_{2 T}^{2}, \mu^{2}\right) d \mathbf{k}_{1 T}^{2} d \mathbf{k}_{2 T}^{2} d y \frac{d \phi_{1}}{2 \pi} \frac{d \phi_{2}}{2 \pi}
\end{aligned}
$$

where $y^{\prime}$ and $y_{g}$ are the rapidities of the final quark or gluon. The analytic expressions for the off-shell matrix elements of subprocesses (1) - (4) has been derived in our previous papers $[12,14]$ (see also [18]). We only mention here that, in accord with the $k_{T}$-factorization prescription $[19,20]$, the off-shell gluon spin density matrix has been taken in the form

$$
\sum \epsilon^{\mu}\left(k_{i}\right) \epsilon^{* \nu}\left(k_{i}\right)=\frac{k_{i T}^{\mu} k_{i T}^{\nu}}{\mathbf{k}_{i T}^{2}}
$$

In all other respects our calculations follow the standard Feynman rules. If we average these expressions over $\phi_{1}$ and $\phi_{2}$ and take the limit $\mathbf{k}_{1 T}^{2} \rightarrow 0$ and $\mathbf{k}_{2 T}^{2} \rightarrow 0$, then we recover the relevant formulas in the leading order collinear approximation of QCD.

\subsection{The KMR unintegrated parton distributions}

In further analysis below we will use the unintegrated quark and gluon densities in a proton which taken in the KMR form [16]. The KMR approach is the formalism to construct the unintegrated parton distributions $f_{a}\left(x, \mathbf{k}_{T}^{2}, \mu^{2}\right)$ from the known conventional parton distributions $x a\left(x, \mu^{2}\right)$, where $a=g$ or $a=q$. In this approximation, the unintegrated quark and gluon distributions are given by $[16]$

$$
\begin{gathered}
f_{q}\left(x, \mathbf{k}_{T}^{2}, \mu^{2}\right)=T_{q}\left(\mathbf{k}_{T}^{2}, \mu^{2}\right) \frac{\alpha_{s}\left(\mathbf{k}_{T}^{2}\right)}{2 \pi} \times \\
\times \int_{x}^{1} d z\left[P_{q q}(z) \frac{x}{z} q\left(\frac{x}{z}, \mathbf{k}_{T}^{2}\right) \Theta(\Delta-z)+P_{q g}(z) \frac{x}{z} g\left(\frac{x}{z}, \mathbf{k}_{T}^{2}\right)\right], \\
f_{g}\left(x, \mathbf{k}_{T}^{2}, \mu^{2}\right)=T_{g}\left(\mathbf{k}_{T}^{2}, \mu^{2}\right) \frac{\alpha_{s}\left(\mathbf{k}_{T}^{2}\right)}{2 \pi} \times \\
\times \int_{x}^{1} d z\left[\sum_{q} P_{g q}(z) \frac{x}{z} q\left(\frac{x}{z}, \mathbf{k}_{T}^{2}\right)+P_{g g}(z) \frac{x}{z} g\left(\frac{x}{z}, \mathbf{k}_{T}^{2}\right) \Theta(\Delta-z)\right],
\end{gathered}
$$

where $P_{a b}(z)$ are the usual unregulated LO DGLAP splitting functions. The theta functions which appear in (10) and (11) imply the angular-ordering constraint $\Delta=\mu /\left(\mu+\left|\mathbf{k}_{T}\right|\right)$ specifically to the last evolution step to regulate the soft gluon singularities. For other evolution steps, the strong ordering in transverse momentum within the DGLAP equations automatically ensures angular ordering 3 . The Sudakov form factors $T_{q}\left(\mathbf{k}_{T}^{2}, \mu^{2}\right)$ and $T_{g}\left(\mathbf{k}_{T}^{2}, \mu^{2}\right)$ which appears in (10) and (11) enable us to include logarithmic loop corrections to the calculated cross sections. The nonlogarithmic corrections can be taken into account by using the $K$-factor $[15,23] K\left(q+\bar{q}^{\prime} \rightarrow W / Z\right) \simeq \exp \left[C_{F} \pi \alpha_{s}\left(\mu^{2}\right) / 2\right]$ with $C_{F}=4 / 3$ and $\mu^{2}=\mathbf{p}_{T}^{4 / 3} m^{2 / 3}$.

\footnotetext{
${ }^{3}$ Numerically, in (10) and (11) we have applied the recent Martin-Stirling-Thorne-Watt (MSTW) LO parametrizations [21] of the collinear parton densities $a\left(x, \mu^{2}\right)$. This choice is differs from the one [12-14] where the Glück-Reya-Vogt (GRV) parton distributions [22] have been used.
} 
The function $f_{q}\left(x, \mathbf{k}_{T}^{2}, \mu^{2}\right)$ in (10) represents the total quark distribution function in a proton. Modifying (10) in such a way that only the first term is kept and the second term omitted, we switch the last gluon splitting off, thus excluding the $f_{q}^{(g)}\left(x, \mathbf{k}_{T}^{2}, \mu^{2}\right)$ component. Taking the difference between the quark and antiquark densities we extract the valence quark component $f_{q}^{(v)}\left(x, \mathbf{k}_{T}^{2}, \mu^{2}\right)=f_{q}\left(x, \mathbf{k}_{T}^{2}, \mu^{2}\right)-f_{\bar{q}}\left(x, \mathbf{k}_{T}^{2}, \mu^{2}\right)$. Finally, keeping only sea quark in first term of (10) we remove the valence quarks from the evolution ladder. In this way only the $f_{q}^{(s)}\left(x, \mathbf{k}_{T}^{2}, \mu^{2}\right)$ contributions to the $f_{q}\left(x, \mathbf{k}_{T}^{2}, \mu^{2}\right)$ are taken into account.

The multidimensional integration in (5) - (8) has been performed by the means of Monte Carlo technique, using the routine VEGAS [24]. The full $\mathrm{C}++$ code is available from the authors on request 4 . This code is practically identical to that used in [12-14].

\section{Numerical results}

We are now in a position to present our numerical results. In all our calculations according to (5) - (8) the light quark masses were set to $m_{u}=4.5 \mathrm{MeV}, m_{d}=8.5 \mathrm{MeV}$ and $m_{s}=155 \mathrm{MeV}$. We have checked that the uncertainties coming from these quantities are negligible compared to the uncertainties connected with the scales in the unintegrated parton densities and strong coupling. We set $m_{c}=1.4 \mathrm{GeV}, m_{W}=80.403 \mathrm{GeV}, m_{Z}=91.1876 \mathrm{GeV}$, $\sin ^{2} \theta_{W}=0.23122$ and use the LO formula for the strong coupling constant $\alpha_{s}\left(\mu^{2}\right)$ with $n_{f}=4$ active quark flavors at $\Lambda_{\mathrm{QCD}}=200 \mathrm{MeV}$ (so that $\alpha_{s}\left(M_{Z}^{2}\right)=0.1232$ ). As it is often done, we choose the renormalization and factorization scales to be equal: $\mu_{R}=\mu_{F}=\mu=m_{T}$, where $m_{T}$ is the transverse mass of the produced vector boson. In the case of prompt photon production we set the scale $\mu$ to be equal to the photon transverse energy $E_{T}^{\gamma}$. We will not study here the scale dependense of our results. This issue is addressed in our previous papers [12-14].

\subsection{Role of the quark contributions}

We begin the discussion by presenting a comparison between the different contributions to the $\gamma / W^{ \pm} / Z^{0}$ cross sections. In Fig. 2 we plot our results for the cross sections as a function of produced photon or gauge boson center-of-mass rapidity $y$. Here, we have performed the calculations for both the proton-antiproton and proton-proton interactions at the Tevatron and LHC energies, respectively. In the case of electroweak boson production, the cross sections are multiplied by the branching fractions $f(W \rightarrow l \nu)$ and $f\left(Z \rightarrow l^{+} l^{-}\right)$. We set these branching fractions to $f(W \rightarrow l \nu)=0.1075$ and $f\left(Z \rightarrow l^{+} l^{-}\right)=0.03366$ [25]. The additional cuts $|y|<2.5$ and $|y|<4$ have been applied in the case of prompt photon production at the Tevatron and LHC. The solid, dashed and dotted histograms in Fig. 2 represent the contributions from the $g^{*}+g^{*} \rightarrow \gamma / W^{ \pm} / Z^{0}+q+\bar{q}^{\prime}, q_{v}+g^{*} \rightarrow \gamma / W^{ \pm} / Z^{0}+q^{\prime}$ and $q_{v}+\bar{q}_{v}^{\prime} \rightarrow W^{ \pm} / Z^{0}$ (or $q_{v}+\bar{q}_{v} \rightarrow \gamma+g$ ) subprocesses, respectively 5 . The thick solid histograms represent the sum of all contributions. One can see that the gluon-gluon fusion is an important photon production mechanism at both the Tevatron and LHC conditions.

\footnotetext{
${ }^{4}$ lipatov@theory.sinp.msu.ru

${ }^{5}$ In the present analysis the contributions from all $2 \rightarrow 1$ subprocesses have been corrected by the factor of $3 / 2$ compared to those from [14]. We thank V.A. Saleev for drawing our attention to this point.
} 
Moreover, it gives a main contribution to the cross section at the LHC. In the case of $W^{ \pm} / Z^{0}$ production, the role of gluon-gluon fusion subprocess is negligible at the Tevatron and is increased greatly at the LHC energy: it contributes only about one or two percent to the

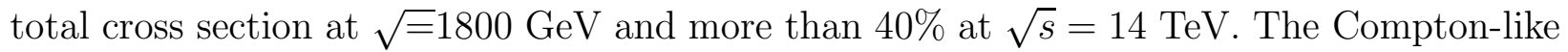
subprocesses $q_{v}+g^{*} \rightarrow \gamma / W^{ \pm} / Z^{0}+q^{\prime}$ are also important.

In Fig. 2, the dash-dotted histograms represent the "reduced sea" component. We find that this component gives approximately 30\% contribution to the total cross section of prompt photon production at the Tevatron and approximately $20 \%$ contribution at the LHC. In the case of electroweak boson production, it contributes about $50 \%$ and $40 \%$, respectively. As it was noted above, this component contains the $q^{(s)}+g^{*} \rightarrow \gamma / W^{ \pm} / Z^{0}+q^{\prime}$, $q^{(s)}+\bar{q}^{(s)} \rightarrow \gamma+g\left(\right.$ or $\left.q^{(s)}+\bar{q}^{(s)} \rightarrow W^{ \pm} / Z^{0}\right)$ and $q^{(s)}+\bar{q}^{(v)} \rightarrow \gamma+g\left(\right.$ or $\left.q^{(s)}+\bar{q}^{(v)} \rightarrow W^{ \pm} / Z^{0}\right)$ subprocesses. The relative contributions are shown in Fig. 3. Note that thick solid histograms in Fig. 3 corresponds to the dash-dotted histograms in Fig. 2. Since all these subprocesses are mainly due to the quarks emerging from the earlier steps of the parton evolution rather than from the last gluon splitting, one can conclude that the quarks constitute an important component of the parton ladder, not negligible even at the LHC energies and not reducible to the gluon component.

\subsection{Comparison with the Tevatron data}

Now we turn to the comparison of our theoretical predictions with the experimental data on the prompt photon and $W^{ \pm} / Z^{0}$ boson production at Tevatron. The data [7-11] on the inclusive prompt photon hadroproduction come from both the $\mathrm{D} \oslash$ and CDF collaborations. The $\mathrm{D} \oslash[7,8]$ data were obtained in the central and forward pseudo-rapidity regions for two different center-of-mass energies, namely $\sqrt{s}=630 \mathrm{GeV}$ and $\sqrt{s}=1800 \mathrm{GeV}$. The central pseudo-rapidity region is defined by the requirement $\left|\eta^{\gamma}\right|<0.9$, and the forward one is defined by $1.6<\left|\eta^{\gamma}\right|<2.5$. The more recent $\mathrm{CDF}$ data $[9,10]$ refer to the same central kinematical region $\left|\eta^{\gamma}\right|<0.9$ for both beam energies $\sqrt{s}=630 \mathrm{GeV}$ and $\sqrt{s}=1800 \mathrm{GeV}$. The data on the electroweak gauge boson production are also come from the $\mathrm{D} \oslash[2-6]$ and CDF [1] collaborations.

The results of our calculations are shown in Figs. $4-9$. Fig. 4 confronts the double differential cross sections $d \sigma / d E_{T} d \eta$ of the prompt photon production calculated at $\sqrt{s}=630$ and $1800 \mathrm{GeV}$ in different kinematical regions with the $\mathrm{D} \oslash[7,8]$ and CDF [9, 10] data. The solid histograms represent calculations in the scheme described above. For comparison, we also show (as dashed histograms) the predictions based on the simple $2 \rightarrow 2$ QCD subprocesses with all quark components summed together. One can see that the both approaches give the very similar results which agree reasonably with the Tevatron data on the prompt photon cross sections within the experimental uncertainties. This fact demonstrates that the high-order corrections for prompt photon production connected with the terms not containing large logarithms are rather small. Our predictions based on the subprocesses (1) (4) are rather similar to ones [26] based on the collinear QCD factorization with the NLO accuracy.

Concerning the electroweak gauge boson production, the situation is slightly different. Figs. 5 and 7 display a comparison between the calculated differential cross sections $d \sigma / d p_{T}$ and the $\mathrm{D} \oslash$ and the CDF experimental data $[1,3,4]$ at low $p_{T}\left(p_{T}<20 \mathrm{GeV}\right)$ and in 
the full $p_{T}$ range. These data have been obtained at $\sqrt{s}=1800 \mathrm{GeV}$. In Figs. 6,8 and 9 , we show the normalized differential cross section $(1 / \sigma) d \sigma / d p_{T}$ and $(1 / \sigma) d \sigma / d|y|$ of the $W^{ \pm}$and $Z^{0}$ boson production in comparison with the data. The predictions based on the scheme (1) — (4) are about a factor of 1.25 higher than the ones based on the simple $2 \rightarrow 1$ subprocesses. The main difference between the predictions is observed in the low $p_{T}$ region. This difference can be attributed to the terms not containing large logarithms and connected with using of the high-order off-shell matrix elements mentioned above. In contrast with the prompt photon production, such terms are significant for the case of $W^{ \pm} / Z^{0}$ production. In [15], an additional factor of about 1.2 was introduced ad hoc to eliminate the visible disagreement between the data and theory. The origin of this extra factor has explained [15] by the fact that the input parton densities (used to determine the unintegrated ones) should themselves be determined from data using the appropriate noncollinear formalism. The results of our calculations based on the scheme (1) - (4) show no need in this extra factor. Some overestimation of our predictions over experimental data at small $p_{T}$ can be, in principle, connected with problem of applicability of BFKL-like evolution in this region and requires an additional study. Note that the traditional QCD calculations (valid in a wide $p_{T}$ range) combine fixed-order perturbation theory (at present, up to NNLO terms [27-31]) with analytic soft-gluon resummation [32,33] and some matching criterion. These calculations gives a similar description of the data [1-6].

Additionally we have studied the effects of the non-logarithmic loop corrections to the gauge boson production amplitude. To do this, we have repeated the calculations based on the $2 \rightarrow 1$ quark-antiquark annihilation with the omitted $K$-factor. The dotted histograms in Figs. $4-9$ correspond to the results of these calculations. We have found a significant (by a factor of about 1.5) reduction of the predicted cross sections. Also, in our numerical calculations we have tested the rather old GRV (LO) parametrizations [22] of the collinear parton distributions in a proton (not shown in plots). We have found that the difference between the results based on the GRV and recent MSTW [21] collinear parton densities is negligible.

Finally, we would like to mention that an additional possibility to distinguish the two calculation schemes comes from studying the ratio of the $W^{ \pm}$and $Z^{0}$ boson cross sections. In fact, since $W^{ \pm}$and $Z^{0}$ production properties are very similar, as the transverse momentum of the vector boson becomes smaller, the radiative corrections affecting the individual distributions and the cross sections of hard process are factorized and cancelled in this ratio. Therefore the results of calculation of this ratio in the scheme (1) - (4) (where the $\mathcal{O}\left(\alpha \alpha_{s}\right)$ and $\mathcal{O}\left(\alpha \alpha_{s}^{2}\right)$ subprocesses are taken into account) and the predictions based on the $\mathcal{O}(\alpha)$ quark-antiquark annihilation should differ from each other at moderate and high $p_{T}$ values. This issue have been studied in our previous paper [14].

\section{Conclusions}

We have studied the production of prompt photon and electroweak gauge bosons in hadronic collisions at high energies in the $k_{T}$-factorization approach of QCD. The central part of our consideration is the off-shell gluon-gluon fusion subprocess $g^{*}+g^{*} \rightarrow \gamma / W^{ \pm} / Z^{0}+q+\bar{q}^{\prime}$. The contribution from the quarks has been taken into account additionally. 
To study the individual contributions from the different partonic subprocesses we have used the KMR scheme. We find that the gluon-gluon fusion is an important production mechanism of prompt photons at both the Tevatron and LHC conditions. At the LHC, it gives the main contribution to the cross section. In the case of $W^{ \pm} / Z^{0}$ production, it contributes only about one or two percent to the total cross section at the Tevatron and more than $20 \%$ at the LHC energy.

We demonstrate that an important contribution to the total cross sections of the processes under consideration also comes from the sea quark interactions. Notably, we found that the contribution of these subprocesses are mainly due to the quarks emerging from the earlier steps of the parton evolution rather than from the last gluon splitting.

\section{Acknowledgements}

We thank S.P. Baranov for participation on the initial stage of investigations, H. Jung, M. Deak and F. Schwennsen for their encouraging interest and very helpful discussions. The authors are very grateful to DESY Directorate for the support in the framework of Moscow - DESY project on Monte-Carlo implementation for HERA - LHC. A.V.L. was supported in part by the grants of the president of Russian Federation (MK-438.2008.2) and Helmholtz - Russia Joint Research Group. Also this research was supported by the FASI of Russian Federation (grant NS-1456.2008.2) and the RFBR fundation (grant 08-02-00896-a).

\section{References}

[1] B. Affolder et al. (CDF Collaboration), Phys. Rev. Lett. 84, 845 (2000).

[2] B. Abbott et al. (Dø Collaboration), Phys. Rev. Lett. 80, 5498 (1998).

[3] B. Abbott et al. (Dø Collaboration), Phys. Rev. D 61, 032004 (2000).

[4] B. Abbott et al. (Dø Collaboration), Phys. Lett. B 513, 292 (2001).

[5] V. Abazov et al. (Dø Collaboration), hep-ex/0702025.

[6] V. Abazov et al. (D Collaboration), arXiv:0712.0803 [hep-ex].

[7] B. Abbott et al. (Dø Collaboration), Phys. Rev. Lett. 84, 2786 (2000).

[8] V.M. Abazov et al. (Dø Collaboration), Phys. Rev. Lett. 87, 251805 (2001).

[9] D. Acosta et al. (CDF Collaboration), Phys. Rev. D 65, 112003 (2002).

[10] D. Acosta et al. (CDF Collaboration), Phys. Rev. D 70, 032001 (2004).

[11] V.M. Abazov et al. (Dø Collaboration), Phys. Lett. 639, 151 (2006).

[12] S.P. Baranov, A.V. Lipatov and N.P Zotov, Phys. Rev. D 77, 074024 (2008).

[13] S.P. Baranov, A.V. Lipatov and N.P Zotov, Eur. Phys. J. C 56, 371 (2008). 
[14] S.P. Baranov, A.V. Lipatov and N.P Zotov, Phys. Rev. D 78, 014025 (2008).

[15] G. Watt, A.D. Martin, and M.G. Ryskin, Phys. Rev. D 70, 014012 (2004).

[16] M.A. Kimber, A.D. Martin, and M.G. Ryskin, Phys. Rev. D63, 114027 (2001);

G. Watt, A.D. Martin, and M.G. Ryskin, Eur. Phys. J. C 31, 73 (2003).

[17] K. Koller, T.F. Walsh, and P.M. Zerwas, Z. Phys. C 2, 197 (1979).

[18] M. Deak and F. Schwennsen, JHEP 0809, 035 (2008).

[19] L.V. Gribov, E.M. Levin, and M.G. Ryskin, Phys. Rep. 100, 1 (1983);

E.M. Levin, M.G. Ryskin, Yu.M. Shabelsky and A.G. Shuvaev, Sov. J. Nucl. Phys. 53, 657 (1991).

[20] S. Catani, M. Ciafoloni and F. Hautmann, Nucl. Phys. B 366, 135 (1991);

J.C. Collins and R.K. Ellis, Nucl. Phys. B 360, 3 (1991).

[21] A.D. Martin, W.J. Stirling, R.S. Thorne, and G. Watt, arXiv:0901.0002 [hep-ph].

[22] M. Glück, E. Reya and A. Vogt, Phys. Rev. D 46, 1973 (1992);

M. Glück, E. Reya and A. Vogt, Z. Phys. C 67, 433 (1995).

[23] A. Kulesza and W.J. Stirling, Nucl. Phys. B 555, 279 (1999).

[24] G.P. Lepage, J. Comput. Phys. 27, 192 (1978).

[25] W.-M. Yao et al. (Particle Data Group), J. Phys. G 33, 1 (2006).

[26] H.-N. Li, Phys. Lett. B 454, 328 (1999);

T. Binoth, J.Ph. Guillet, E. Pilon, and M. Werlen, Eur. Phys. J. C 16, 311 (2000);

S. Catani, M. Fontannaz, J.Ph. Guillet, and E. Pilon, JHEP 05, 028 (2002);

L.E. Gordon and W. Vogelsang, Phys. Rev. D 48, 3136 (1993).

[27] P. Sutton, A. Martin, R. Roberts, and W.J. Stirling, Phys. Rev. D 45, 2349 (1992).

[28] R. Rijken and W. van Neerven, Phys. Rev. D 51, 44 (1995).

[29] R. Hamberg, W. van Neerven, and T. Matsuura, Nucl. Phys. B 359, 343 (1991).

[30] R. Harlander and W. Kilgore, Phys. Rev. Lett. 88, 201801 (2002).

[31] W. van Neerven and E. Zijstra, Nucl. Phys. B 382, 11 (1992).

[32] R.K. Ellis and S. Veseli, Nucl. Phys. B 511, 649 (1998).

[33] C. Balazs and C.P. Yuan, Phys. Rev. D 56, 5558 (1997). 


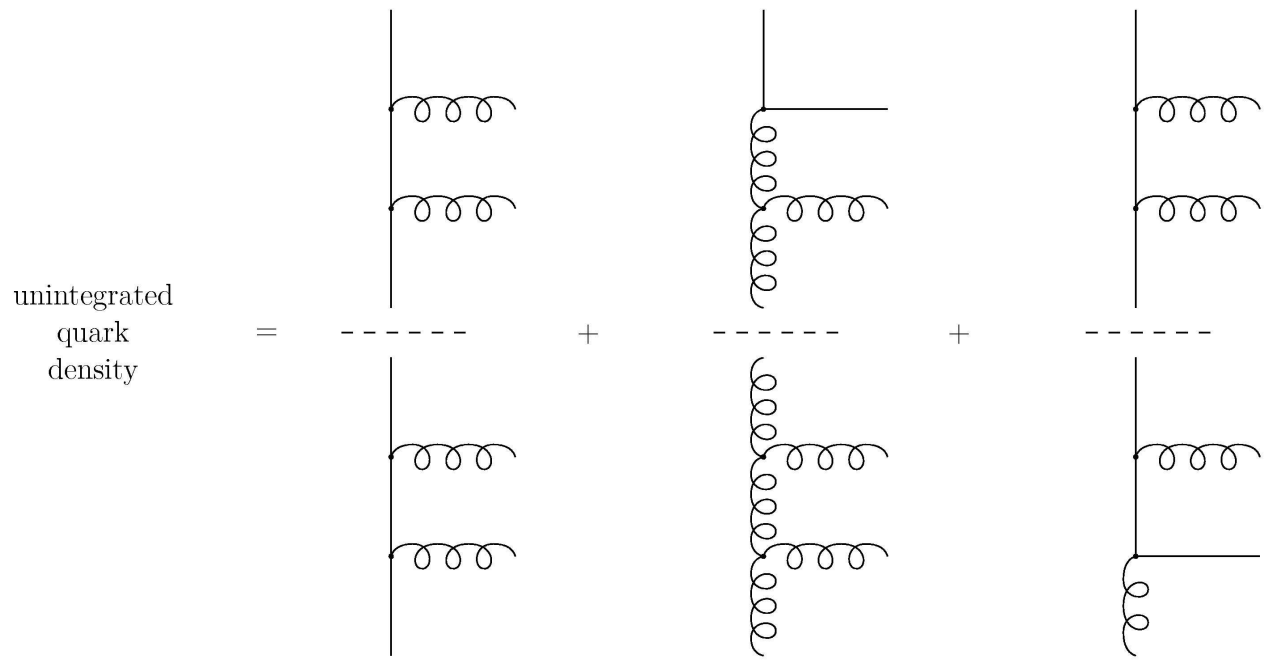

Figure 1: Our approach to calculate the unintegrated quark distributions. 

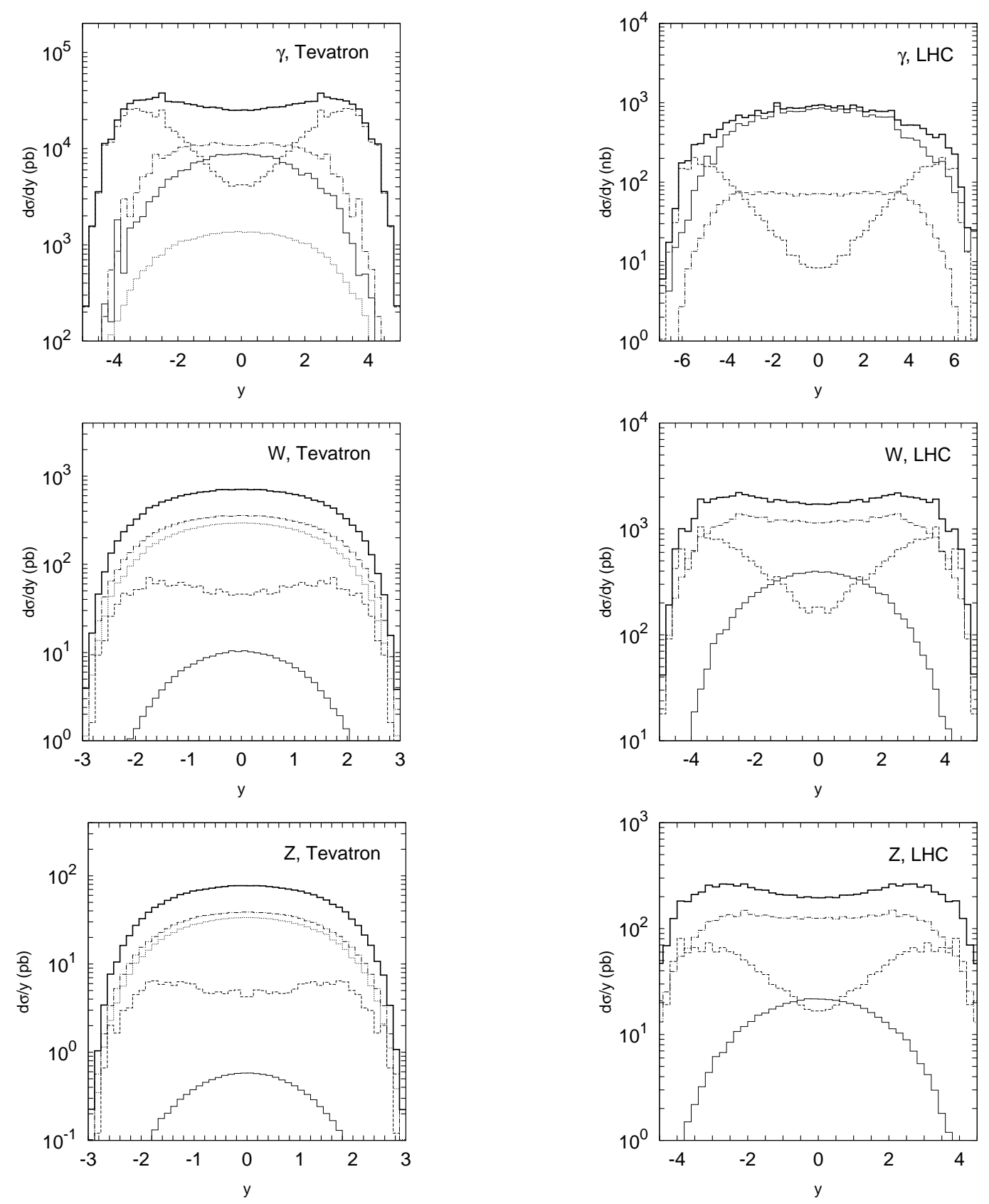

Figure 2: Differential cross sections of prompt photon or electroweak boson production at the Tevatron and LHC as a function of their c.m. rapidity $y$. The solid, dashed and dotted histograms represent the contributions from the $g^{*}+g^{*} \rightarrow \gamma / W^{ \pm} / Z^{0}+q+\bar{q}^{\prime}, q_{v}+g^{*} \rightarrow$ $\gamma / W^{ \pm} / Z^{0}+q^{\prime}$ and $q_{v}+\bar{q}_{v} \rightarrow \gamma+g$ (or $q_{v}+\bar{q}_{v} \rightarrow W^{ \pm} / Z^{0}$ ) subprocesses, respectively. The dash-dotted histograms represent the "reduced sea" component. The thick solid histograms represent the sum of all contributions. 

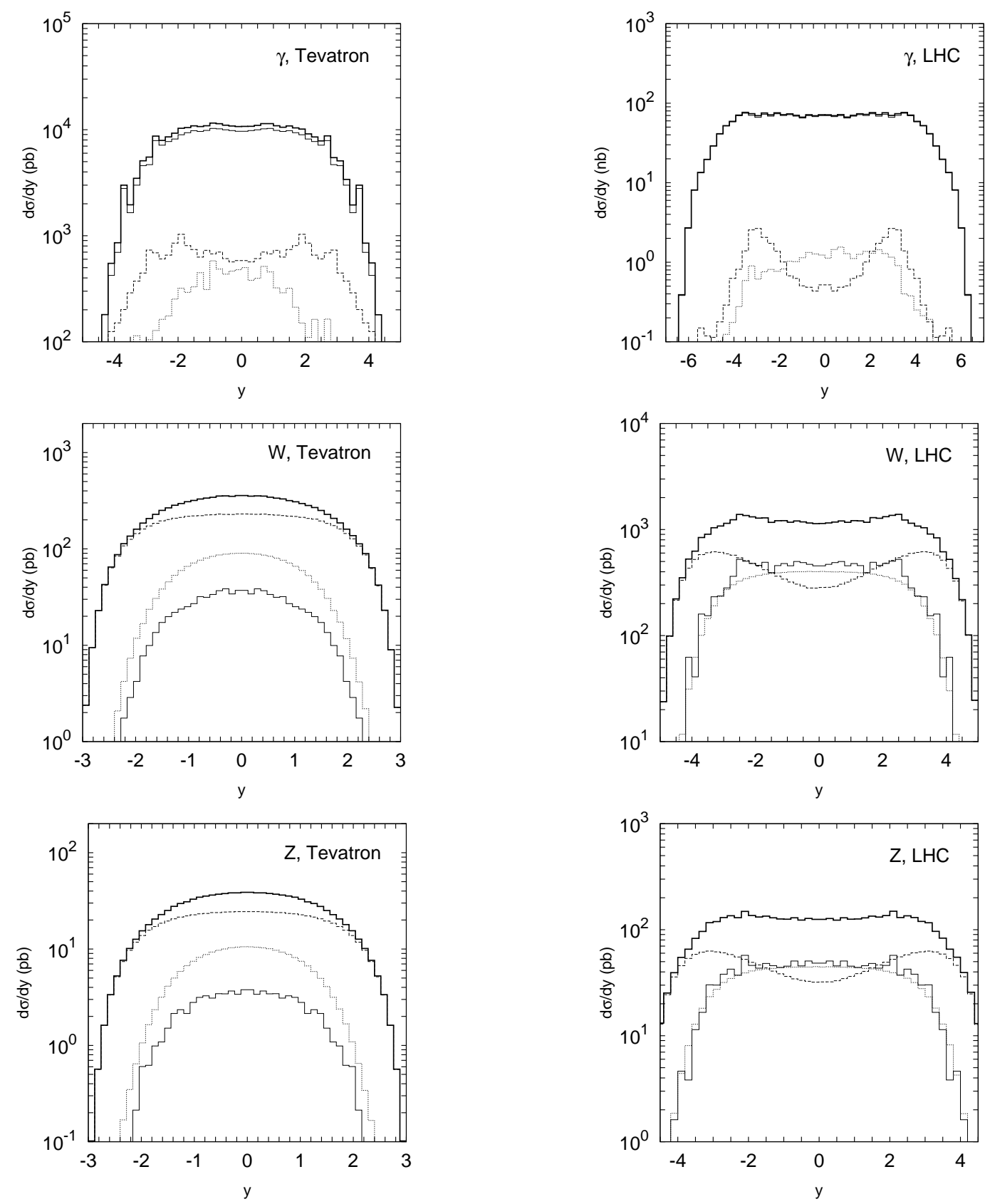

Figure 3: Different contributions from the "reduced sea" component to the prompt photon or electroweak boson production cross sections at the Tevatron and LHC. The solid, dashed and dotted histograms represent the contributions from the $q_{s}+g^{*} \rightarrow \gamma / W^{ \pm} / Z^{0}+q^{\prime}, q_{s}+\bar{q}_{v} \rightarrow$ $\gamma+g$ (or $q_{s}+\bar{q}_{v}^{\prime} \rightarrow W^{ \pm}$) and $q_{s}+\bar{q}_{s} \rightarrow \gamma / W^{ \pm} / Z^{0}+g$ subprocesses, respectively. The thick solid histograms represent the sum of all contributions. 
a)

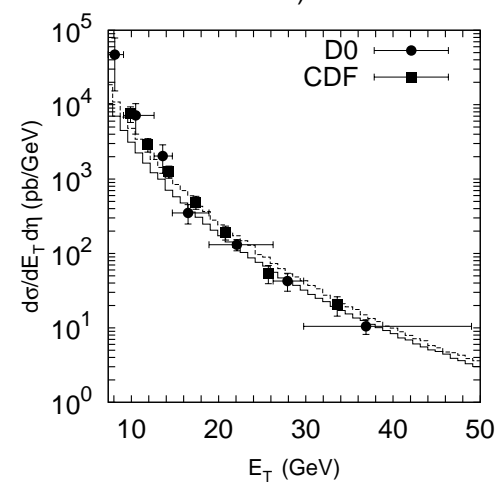

c)

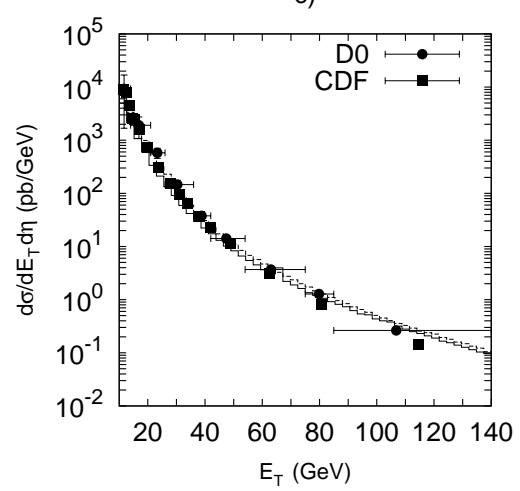

b)

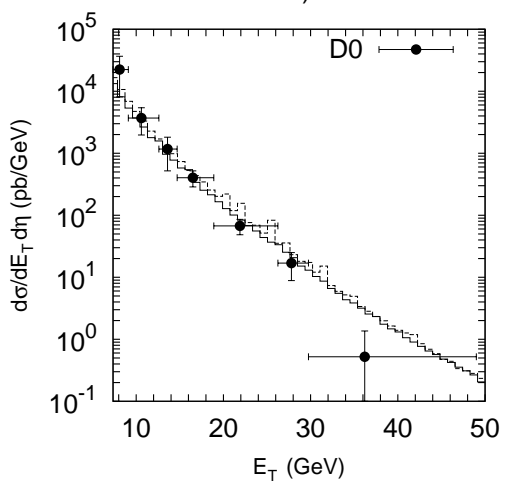

d)

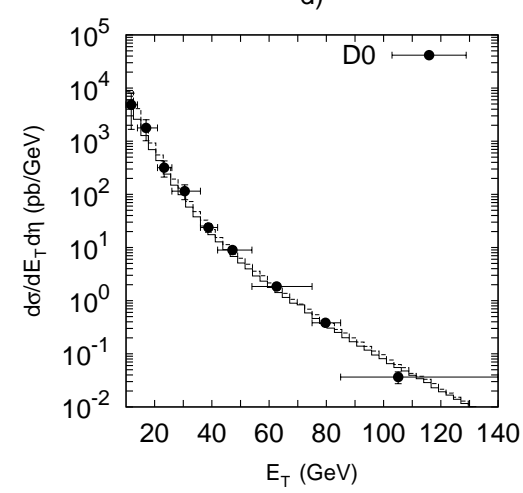

Figure 4: Double differential cross section $d \sigma / d E_{T} d \eta$ for the inclusive prompt photon hadroproduction calculated for $|\eta|<0.9$ (a) and $1.6<|\eta|<2.5$ (b) at $\sqrt{s}=630 \mathrm{GeV}$ and for $|\eta|<0.9$ (c) and $1.6<|\eta|<2.5$ (d) at $\sqrt{s}=1800 \mathrm{GeV}$. Solid histograms represent calculations in the "decomposition" scheme where all contributions described in the text are taken into account. Dashed histograms correspond to the predictions based on the simple $2 \rightarrow 2$ quark-gluon QCD interaction and quark-antiquark annihilation subprocess with all quark components summed together. The experimental data are from $\mathrm{D} \oslash[7,8]$ and $\mathrm{CDF}[9$, 10]. 

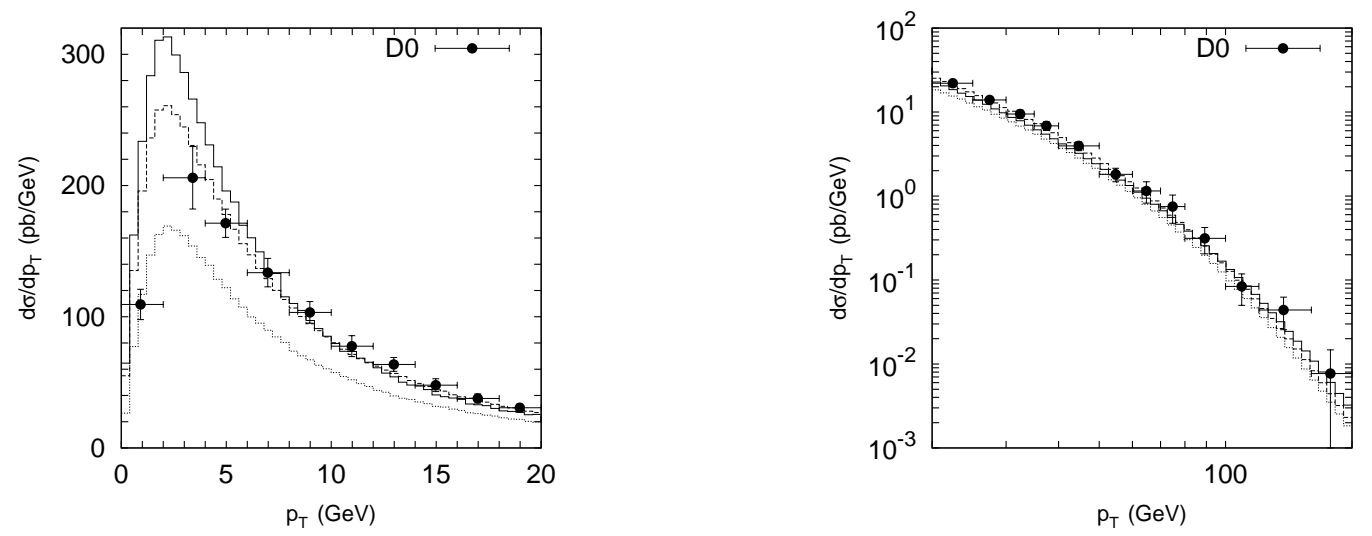

Figure 5: Transverse momentum distribution of the $W^{ \pm}$boson production calculated at $\sqrt{s}=1800 \mathrm{GeV}$. Solid histograms represent calculations in the "decomposition" scheme where all contributions described in the text are taken into account. Dashed histograms correspond to the predictions based on the simple $2 \rightarrow 1$ quark-antiquark annihilation subprocess with all quark components summed together. Dotted histograms correspond to the simple $2 \rightarrow 1$ quark-antiquark annihilation subprocess without $K$-factor. The cross sections time branching fraction $f(W \rightarrow l \nu)$ are shown. The experimental data are from $\mathrm{D} \oslash[4]$.
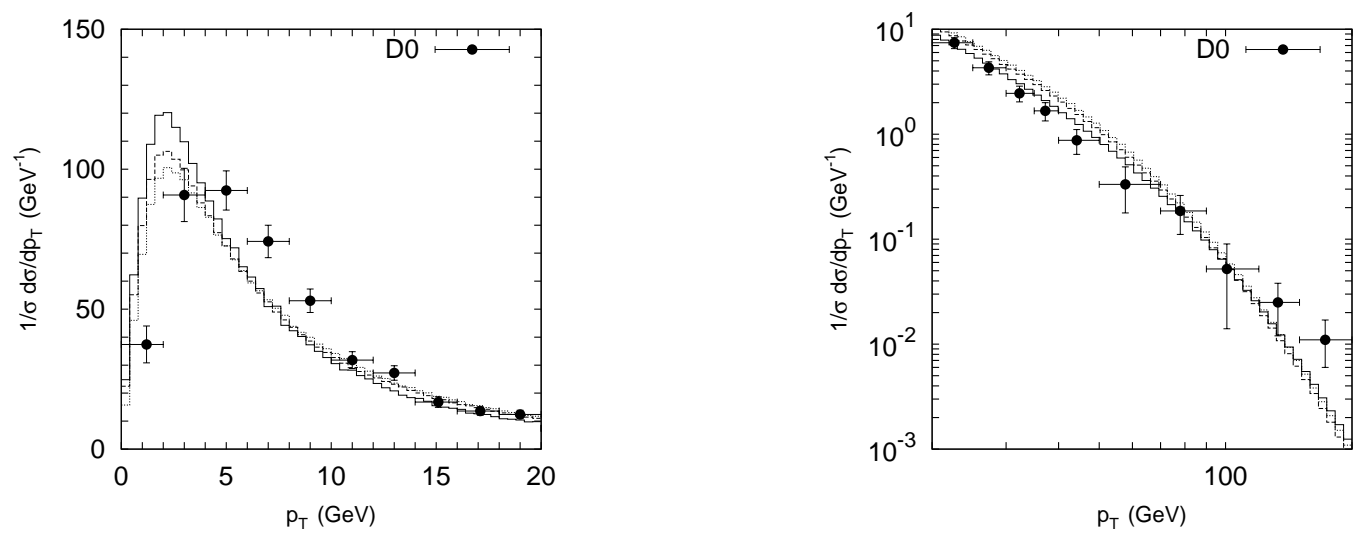

Figure 6: Normalized transverse momentum distribution of the $W^{ \pm}$boson production calculated at $\sqrt{s}=1800 \mathrm{GeV}$. Notation of the histograms is the same as in Fig. 5. The experimental data are from $\mathrm{D} \oslash[2]$. 

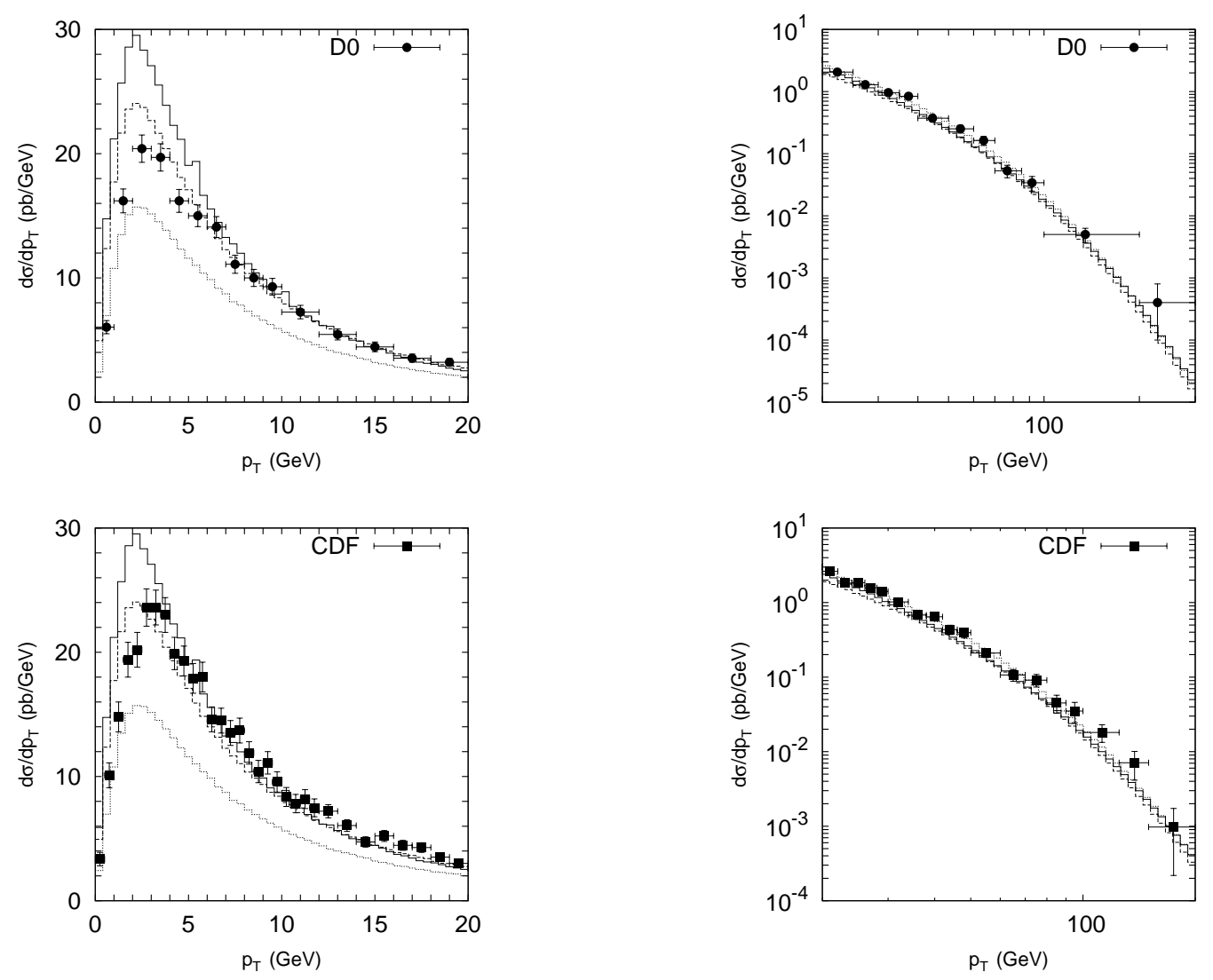

Figure 7: Transverse momentum distribution of the $Z^{0}$ boson production calculated at $\sqrt{s}=1800 \mathrm{GeV}$. Notation of the histograms is the same as in Fig. 5. The cross sections time branching fraction $f\left(Z \rightarrow l^{+} l^{-}\right)$are shown. The experimental data are from $\mathrm{D} \oslash[3]$ and $\mathrm{CDF}[1]$.
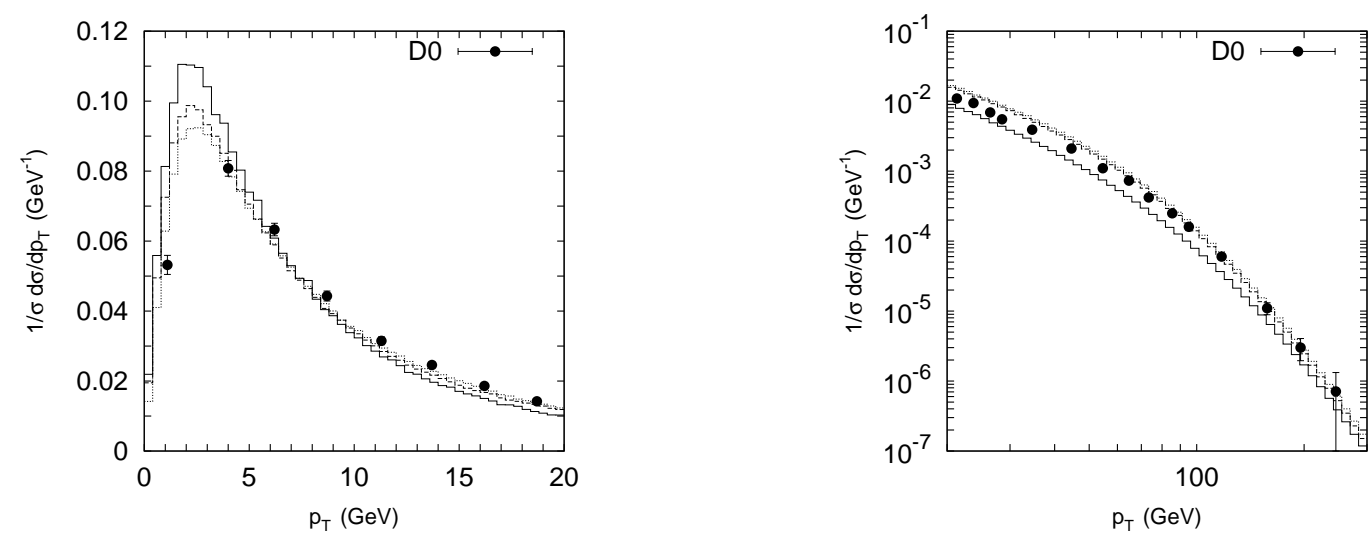

Figure 8: Normalized transverse momentum distribution of the $Z^{0}$ boson production calculated at $\sqrt{s}=1960 \mathrm{GeV}$. Notation of the histograms is the same as in Fig. 5. The experimental data are from $\mathrm{D} \oslash[6]$. 


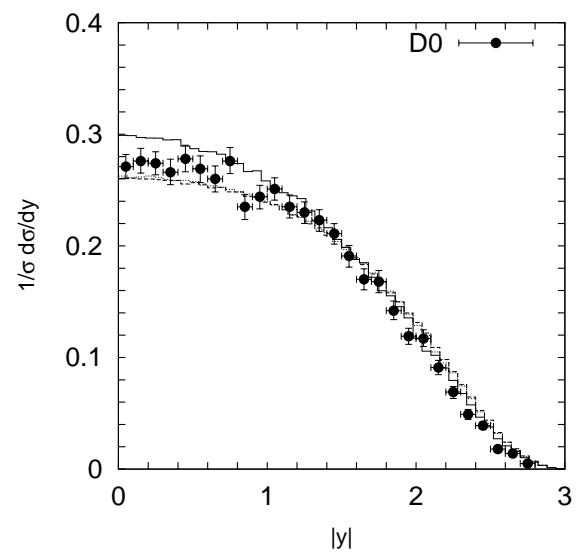

Figure 9: Normalized rapidity distribution of the $Z^{0}$ boson production calculated at $\sqrt{s}=$ $1960 \mathrm{GeV}$. Notation of the histograms is the same as in Fig. 5. The experimental data are from $\mathrm{D} \oslash[5]$. 